The ultimate fate of the sling cells remains a mystery. It was previously thought that they underwent programmed cell death. However, although Shu et al. identified a few apoptotic cells in the sling just before birth, the extent of cell death was insufficient to account for its disappearance. The authors therefore make the tantalizing suggestion that the sling neurons might survive and migrate to other regions of the brain. The question of where the cells go, and what contribution, if any, they make to the adult brain, should provide ample scope for future study.

Heather Wood

\section{(9) References and links} ORIGINAL RESEARCH PAPER Shu, T. et al. The glial sling is a migratory population of developing neurons. Development 130, 2929-2937 (2003) FURTHER READING Silver, J. et al. Axonal guidance during development of the great cerebral commissures: descriptive and experimental studies, in vivo, of the role of preformed glial pathways. J. Comp. Neurol. 210 10-29 (1982)

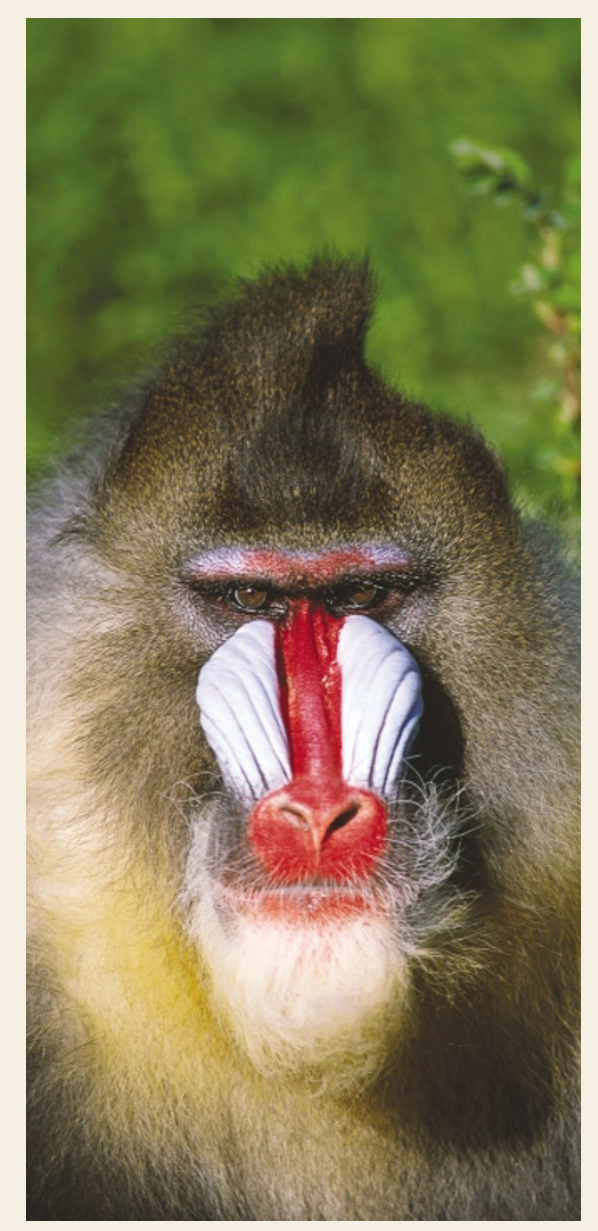

NEURODEGENERATIVE DISEASE

\title{
Ataxin the brain
}

Polyglutamine diseases - presently untreatable human neurodegenerative disorders that include Huntington's disease and several types of spinocerebellar ataxia (SCA) - are associated with expansion of CAG trinucleotide repeats. In SCA type 1, elevated polyglutamine concentration occurs in the kinase ataxin- 1 , and accumulation of this protein is often seen in the neuronal nuclei. Now, reports published in 8 May issue of Neuron and 16 May issue of Cell demonstrate that phosphorylation of ataxin-1 plays a crucial role in SCA type 1 pathogenesis.

In neurons of SCA1 transgenic mice, a mutated gene encoding ataxin- 1 directs production of the protein with an increasing number of consecutive residues of the amino acid glutamine, which leads to the formation of nuclear inclusions. Although the glutamine repeats certainly contribute to protein misfolding and disease progression, recent research has indicated that key interactions between ataxin-1 and other proteins could involve residues of ataxin- 1 other than the polyglutamine tract.

Teams led by H. Orr and H. Zoghbi assessed whether phosphorylation has a role in regulating the function and pathogenicity of ataxin-1. They report in Neuron that one specific amino acid of ataxin- 1 - serine 776 - is a site of phosphorylation. The removal of the phosphorylation site affected the cellular deposition of polyglutamine-expanded ataxin-1, which then failed to form nuclear inclusions in tissue culture cells. When this form of ataxin- 1 was expressed in mice, even though the protein was found to be expressed within the neuronal nuclei, the mice exhibited a marked decrease in neuronal dysfunction. This indicates that phosphorylation of the specific serine residue on ataxin-1 plays a crucial role in modulating the ability of the mutant form of ataxin-1 to induce neurodegeneration by influencing its normal interactions with other proteins and the formation of nuclear inclusions.

In the Cell paper, the researchers discovered that a multifunctional regulatory molecule, the 14-3-3 protein, mediates the neurotoxicity of ataxin- 1 by binding to and stabilizing the protein, thereby slowing its normal degradation. The further finding that the association of ataxin-1 with 14-3-3 is regulated by phosphorylation of another regulatory protein, Akt, and that in a Drosophila model of SCA1 both 14-3-3 and Akt modulate neurodegeneration, provides further insight into SCA1 pathogenesis and identifies potential targets for therapeutic intervention.

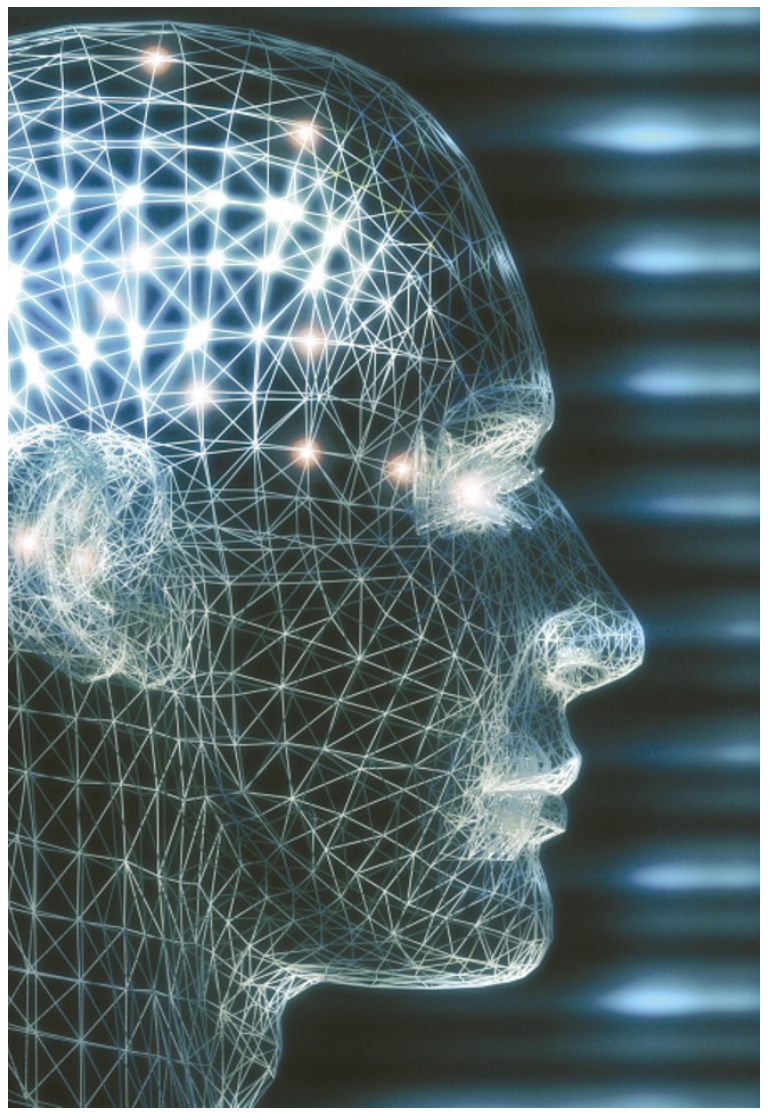

This body of work shows that expansion of the polyglutamine tract or nuclear expression alone are not sufficient to cause disease, but that phosphorylation of ataxin-1 contributes to disease progression. The proteins with which ataxin-1 normally interacts are likely to be important in the disease process, indicating that blocking the phosphorylation events could be a viable treatment. This is particularly exciting because kinases are tractable targets in drug discovery. Melanie Brazil, Senior Editor, Nature Reviews Drug Discovery

(2) References and links ORIGINAL RESEARCH PAPERS Emamiam, E. S. et al. Serine 776 of ataxin- 1 is critical for polyglutamine-induced disease in SCA1 transgenic mice. Neuron 38, 375-387 (2003) | Chen, H.-K. et al. Interaction of Akt-phosphorylated ataxin-1 with 14-3-3 mediates neurodegeneration in spinocerebellar ataxia type 1. Cell 113, 457-468 (2003)

FURTHER READING Gusella, J. F. \& MacDonald, M. E. Molecular genetics: unmasking polyglutamine triggers in neurodegenerative disease. Nature Rev. Neurosci. 1, 109-115 (2000)| Orr. H. T. \& Zoghbi, H. Y. SCA1 molecular genetics: a history of a 13-year collaboration against glutamines. Hum. Mol. Genet. 10, 2307-2311 (2001) | Orr, H. T. The ins and outs of a polyglutamine neurodegenerative disease: spinocerebellar ataxia type 1 (SCA1). Neurobiol. Dis. 7, 129-134 (2000) 